\title{
Editorial
}

\section{eCAM: Attacking an Epidemic?}

\section{Edwin L. Cooper}

Laboratory of Comparative Neuroimmunology, Department of Neurobiology, David Geffen School of Medicine at UCLA, University of California, Los Angeles, CA 90095-1763, USA

Corresponding author: Edwin L. Cooper, Laboratory of Comparative Neuroimmunology, Department of Neurobiology, David Geffen School Of Medicine at UCLA, University of California, Los Angeles, CA 90095-1763, USA. Tel: (310) 825-9567; Fax: (310) 825-2224; E-mail: ecam@mednet.ucla.edu

Diabetes 2, once reserved for those who are aging, is now rampant in adolescents in the USA. Obesity is the culprit brought on by poor eating habits, lack of exercise and a general malaise paradoxically due to wealth and not poverty (at least material). In our penultimate issue of $e C A M$ supported by OUP, I aim to show certain advancements in evidence-based approaches. Diabetes is a condition in persons who have a high blood sugar (glucose) level either because the body does not produce enough insulin or because body cells do not properly respond to the insulin that is produced. Insulin is a hormone produced in the pancreas which enables body cells to absorb glucose, and to convert into energy. If the body cells do not absorb glucose, the glucose accumulates in the blood (hyperglycemia), leading to vascular, nerve and other complications. Serious long-term complications include cardiovascular disease, chronic renal failure and retinal damage. Adequate treatment of diabetes is thus important as well as blood pressure control and lifestyle factors such as smoking cessation and maintaining a healthy body weight. As of 2000, at least 171 million people worldwide suffer from diabetes, or $2.8 \%$ of the population. Type 2 diabetes is by far the most common, affecting $90-95 \%$ of the US's diabetes population.

Guo and colleagues (1) propose a hypothesis. They suggest one novel vanadium complex of vanadium-enriched Cordyceps sinensis (VECS), which is beneficial in preventing depression in diabetes and it can influence the longterm course of glycemic control. Vanadium compounds imitate the action of insulin, and this mimicry may have further favorable effects on levels of treatment satisfaction and mood. C. sinensis has an antidepressant-like activity, and attenuates the diabetes-induced increase in blood glucose concentrations. Thus, they suggest that the VECS may be a potential strategy for a more contemporary treatment of depression and diabetes through the co-effect of $C$. sinensis and vanadium. In an animal model, to test the validity of this hypothesis, investigators can examine blood glucose levels, which in turn are measured by swimming and climbing behavior in streptozotocin-induced hyperglycemic rats. This strongly supports the use of animal models to help understand disease.

In contrast to the basic science approach, Aljasir et al. (2) have reviewed a clinical strategy, and that is Yoga practice for the management of diabetes in adults. All randomized controlled clinical trials comparing yoga practice with other types of intervention or with regular practice or both were examined. Two independent reviewers assessed each study for quality. The mean difference was used for summarizing the effect of each study outcome with $95 \%$ confidence intervals. Results show improvement in outcomes among patients with diabetes but these were among short-term or immediate diabetes outcomes; not all were statistically significant, leaving inconclusive results and insignificant long-term outcomes. Therefore no adverse effects were reported in any of the studies. Short-term benefits for patients with diabetes may be achieved by practicing yoga. Further research is needed in this area. Factors like quality of the trials and other methodological concerns should be improved by large randomized control trials with allocation concealment to assess the effectiveness of yoga on diabetes. Although promising, definitive recommendations for 
physicians to encourage their patients to practice yoga are not yet conclusive. We need more evidence-based approaches.

As a basic science approach, Choi et al. (3) analyzed diabetic nephropathy - one of the most frequent and serious complications of diabetes. Soybeans have been shown to reduce urinary albumin excretion and total cholesterol in non-diabetic patients with nephrotic syndrome. However, because reports focusing specifically on diabetic nephropathy are scarce and the available results are inconsistent, soybean consumption was found to reduce urinary protein excretion in type 1 diabetic patients with diabetic nephropathy, whereas it elicited an increase in urinary protein excretion when soybeans were consumed by type 2 diabetic patients. Again, animal models are advantageous. Male Sprague-Dawley rats were assigned to one of three groups: control, diabetic with red chow diet and diabetic with soybean diet. For histological examination, the expression of osteopontin (OPN) and aquaporin (AQP), renal function and hemoglobin A1c were evaluated at the end of the study. Improvements in glomerular and tubulointerstitial lesions were demonstrated in the diabetic rat group given a soybean diet. OPN Costeopontiu and AQP aquaporin expression were suppressed in the kidneys of diabetic rats that consumed the soybean diet. With these results, a tentative conclusion is proposed. Soybeans may prevent weight loss and morphological disruption of the kidney and may also improve glycemic control. Thus long-term control of blood glucose levels using a soybean diet could prevent the progression of diabetes, and therefore, nephropathy might be prevented." (3).

Finally another clinical approach shows promise. "Satureja khuzestanica is an endemic plant widely distributed in the Southern part of Iran. It has antioxidant properties and seems to be useful in diseases related to oxidative stress such as diabetes and hyperlipidemia. As a test, they investigated the effect of $S$. khuzestanica supplement on metabolic parameters of hyperlipidemic patients with diabetes. Twenty-one hyperlipidemic patients with diabetes were randomized in a double blind, placebo-controlled clinical trial to receive either S. khuzestanica (tablets contain $250 \mathrm{mg}$ dried leaves) or placebo once a day for 60 days. Blood samples were obtained at baseline at the end. Samples were analyzed for levels of glucose, total cholesterol, LDL-cholesterol, HDL-cholesterol, triglyceride, creatinine, thiobarbituric acid reactive substances (TBARS) as marker of lipid peroxidation and ferric reducing ability [total antioxidant power, (TAP)]. Treatment of patients by S. khuzestanica for 60 days induced significant decrease in total cholesterol $(P=0.008)$ and LDL-cholesterol $(P=0.03)$ while increased HDL-cholesterol $(P=0.02)$ and TAP $(P=0.007)$ in comparison with the baseline values. S. khuzestanica did not alter blood glucose, triglyceride, creatinin and TBARS levels. In comparison with baseline values, no significant change was observed in blood glucose, total cholesterol, LDL-cholesterol, HDLcholesterol, triglyceride, creatinine, TBARS and TAP in placebo-treated group. They therefore recommended the usage of $S$. khuzestanica as a supplement to drug regimen of diabetic type 2 patients with hyperlipidemia" (4).

These four approaches to a growing health problem of enormous proportions have reached an avenue for providing evidence-based results. $e C A M$ will remain committed to critical thinking and strategies that will move us closer to self awareness, personal commitment and control. These two points that fuel research should, in the next few years, offer some relief to this paradoxical condition fueled by personal negligence.

\section{References}

1. Guo JY, Han CC, Liu YM. A contemporary treatment approach to both diabetes and depression by Cordyceps sinensis, rich in Vanadium. eCAM November 30, 2009; doi: 10.1093/ecam/nep201 [Epub ahead of print].

2. Aljasir B, Bryson M, Al-Shehri B. Yoga practice for the management of type II diabetes mellitus in adults: a systematic review. eCAM May 7, 2008; doi: 10.1093/ecam/nen027 [Epub ahead of print].

3. Choi YE, Ahn SK, Lee WT, Lee JE, Park SH, Yoon BB, et al. Soybeans ameliolate diabetic nephropathy in rats. eCAM March 20, 2008; doi: 10.1093/ecam/nen021 [Epub ahead of print].

4. Vosough-Ghanbari S, Rahimo R, Khrarabaf S, Zeinali S, Mohammadirad A, Amini S, et al. Effects of Satureja khuzestanica on serum glucose, lipids and markers of oxidative stress in patients with type 2 diabetes mellitus: a double-blind randomized controlled trial. eCAM February 27, 2008; doi: 10.1093/ecam/nen018 [Epub ahead of print]. 


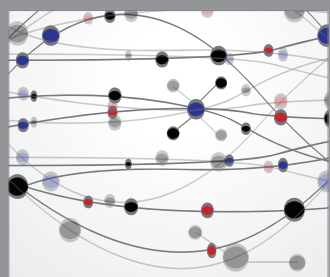

The Scientific World Journal
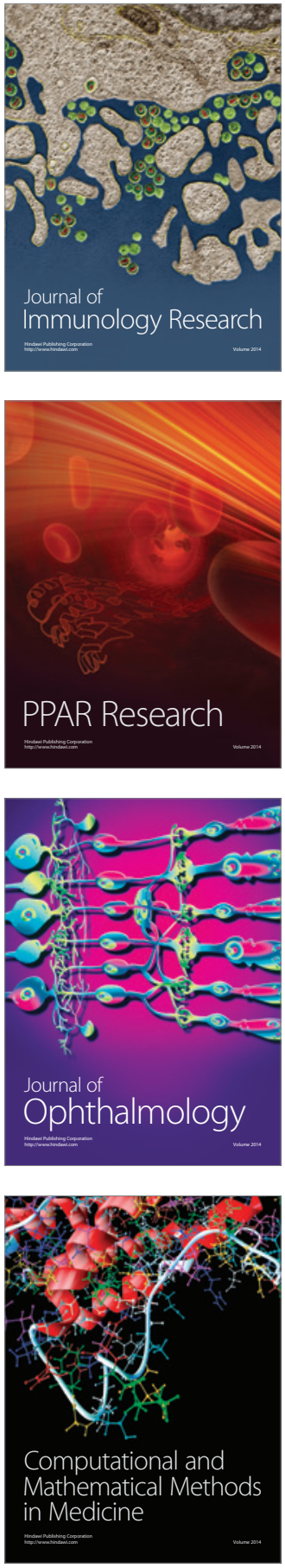

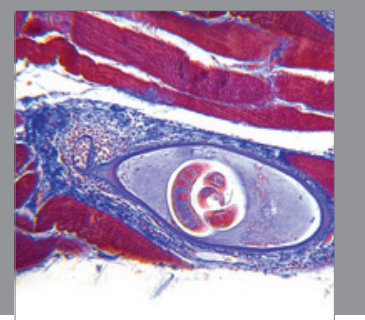

Gastroenterology

Research and Practice
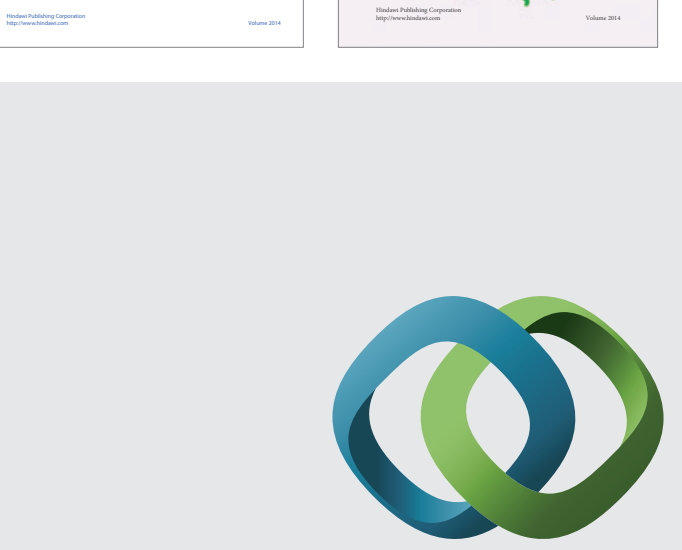

\section{Hindawi}

Submit your manuscripts at

http://www.hindawi.com
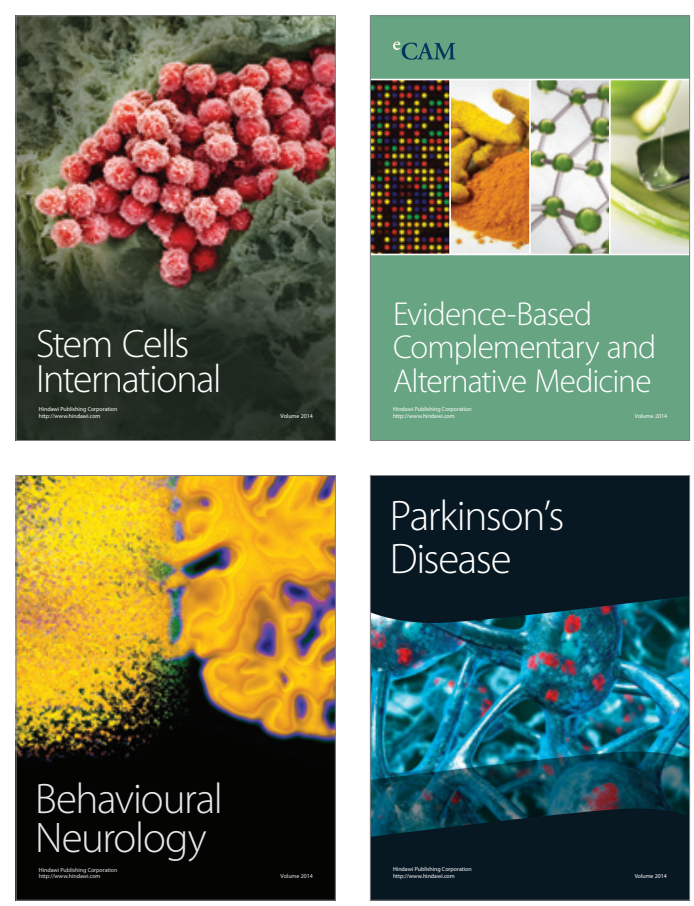

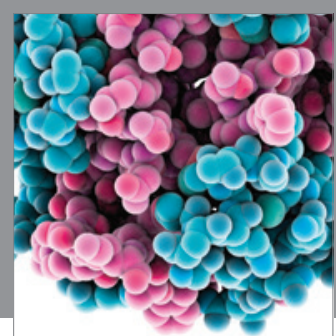

Journal of
Diabetes Research

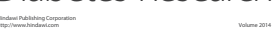

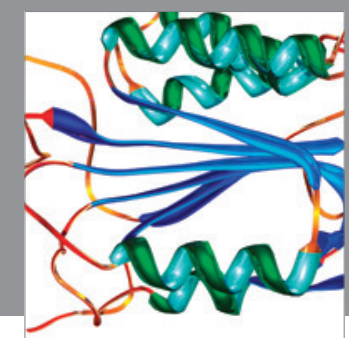

Disease Markers
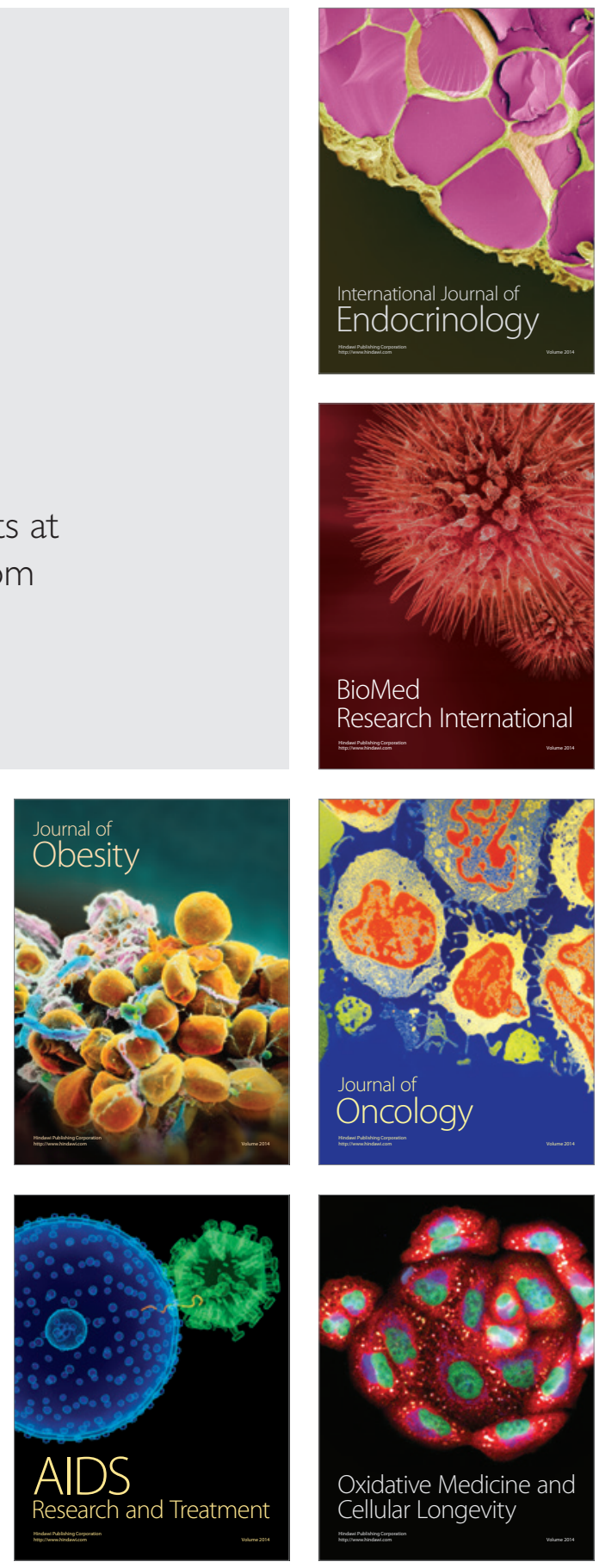